\title{
BILINEAR FUNCTIONALS ON THE SPACE OF BOUNDED, MEASURABLE FUNCTIONS
}

\section{BY H. H. GOLDSTINE}

1. Introduction. We propose to make an extension of the Radon integral to two variables, and by properly defining the notion of limited variation to show that every continuous bilinear functional on the class of bounded, measurable functions (with suitably defined norm) is expressible in terms of this integral. Conversely we show that the integral is bilinear and continuous. The paper is concluded with a theorem establishing a relation between the double and iterated integrals. Similar results for the case of continuous linear functionals have recently been obtained.*

2. Preliminary Definitions. Let $\subseteq$ be the class of all measurable subsets of the interval $\Im=[0 \leqq x \leqq 1]$, and let $\phi$ be a function on $\mathfrak{S}$ s to $\mathfrak{A}$, the set of all real numbers. Such a function will be termed $b i$-additive in case, for every two disjoint sets $\sigma_{1}, \sigma_{2}$, we have $\phi\left(\sigma, \sigma_{1}+\sigma_{2}\right)=\phi\left(\sigma, \sigma_{1}\right)+\phi\left(\sigma, \sigma_{2}\right)$ and $\phi\left(\sigma_{1}+\sigma_{2}, \sigma\right)$ $=\phi\left(\sigma_{1}, \sigma\right)+\phi\left(\sigma_{2}, \sigma\right)$; the function $\phi$ will be said to be of limited variation in case the upper bound of

$$
\left|\sum_{i, j} \epsilon_{1 i} \phi\left(\sigma_{1 i}, \sigma_{2 j}\right) \epsilon_{2 j}\right|
$$

for all divisions $\sigma_{k_{1}}, \cdots, \sigma_{k m_{k}},(k=1,2)$, of $\Im$ into disjoint sets and all numbers $\epsilon_{k i}$ such that $\left|\epsilon_{k i}\right|=1,(k=1,2 ; i=1,2, \cdots$, $m_{k}$ ), is finite; $\dagger$ we shall designate this bound, when it is finite, by $T \phi$.

Let $\mu_{1}, \mu_{2}$ be any two functions in $\mathfrak{M}$, the class of bounded, measurable functions, such that $d_{k 0} \leqq \mu_{k}(x)<D_{k}, \quad(k=1,2$; $0 \leqq x \leqq 1)$. Then following the usual procedure in the Lebesgue

* See, T. H. Hildebrandt, On bounded linear functional operations, Transactions of this Society, vol. 36 (1934), p. 868; or G. Fichtenholz and L. Kantorovitch, Sur les opérations dans l'espace des fonctions bornées, Studia Mathematica, vol. 5 (1934), p. 69.

$\dagger$ This definition is a generalization to set functions of Fréchet's definition for functions of real variables; see, M. Fréchet, Sur les fonctionelles bilinéaires, Transactions of this Society, vol. 16 (1915), p. 215. 
theory, we divide $\left(d_{k 0}, D_{k}\right)$ into sub-intervals by numbers $d_{k 0}<d_{k 1}<\cdots<d_{k m_{k}}=D_{k}, \quad(k=1,2)$; this process gives rise to a partition $\pi$ of the rectangle determined by $d_{k 0}$ and $D_{k}$. Throughout this paper we shall use the term partition in this restricted sense. To simplify further the notation let $\sigma_{k i}$ be the set of all $x$ such that $d_{k i} \leqq \mu_{k}(x)<d_{k i+1}$. Finally if the limit, as the norm of $\pi$ approaches zero, of

$$
s(\pi)=\sum_{\pi} \xi_{1 i} \xi_{2 j} \phi\left(\sigma_{1 i}, \sigma_{2 j}\right)
$$

exists, where $d_{k i} \leqq \xi_{k i} \leqq d_{k i+1}$, it will be designated by

$$
\int_{0}^{1} \int_{0}^{1} \mu_{1}(s) \mu_{2}(t) \phi(d \Im, d \Im) .
$$

3. The Existence of the Integral. We proceed to a statement of a sufficient condition for the existence of the integral.

THEOREM 1. If the set function $\phi$ on $\subseteq \subseteq$ to $\mathfrak{A}$ is of limited variation and bi-additive, the double integral (3) exists for every pair $\mu_{1}$, $\mu_{2}$ of functions in $\mathfrak{M}$.

The proof of this theorem follows the usual lines of demonstration for the existence of a Lebesgue integral of a bounded function.

We shall associate with the class $\mathfrak{M}$ two norms \|\|$_{1}$ and \|\|$_{2}$ which will thus give rise to two normed linear spaces $\mathfrak{M}_{k}$ $=\left(\mathfrak{M},\|\|_{k}\right),(k=1,2)$. These norms are defined as follows: ${ }^{*}$

$$
\|\mu\|_{1} \equiv \underset{i}{\bar{B}}|\mu(t)|
$$

and

$$
\|\mu\|_{2} \equiv \underset{0 \leqq t \leqq 1}{\operatorname{true} \max }|\mu(t)|=\lim _{n=\infty}\left[\int_{0}^{1}|\mu(t)|^{n} d t\right]^{1 / n} .
$$

4. The Representation Theorem. It should be noted that if a functional $K$ is bilinear and continuous on $\mathfrak{M}_{2} \mathfrak{M}_{2}$, it also has these properties as on $\mathfrak{M}_{1} \mathfrak{M}_{1}$.

THEOREM 2. If $K$ on $\mathfrak{M}_{1} \mathfrak{M}_{1}$ to $\mathfrak{A}$ is bilinear and continuous, then there exists a function $\phi$ on $\subseteq \mathfrak{S}$ to $\mathfrak{A}$ which is bi-additive and of limited variation such that

* See Fichtenholz and Kantorovitch, loc. cit., p. 69. 


$$
K\left(\mu_{1}, \mu_{2}\right)=\int_{0}^{1} \int_{0}^{1} \mu_{1}(s) \mu_{2}(t) \phi(d \Im, d \Im),
$$

for every $\mu_{1}, \mu_{2}$ in $\mathfrak{M}_{1}$; moreover the modulus of $K$ is the total variation Th of $\phi$. If $K$ is bilinear and continuous on $\mathfrak{M}_{2} \mathfrak{M}_{2}$, then $\phi$ has the additional property that the measure of $\sigma_{1}$ or $\sigma_{2}=0$ implies that $\phi\left(\sigma_{1}, \sigma_{2}\right)=0$.

Indicating, as before, the characteristic function of a set $\sigma$ by $\mu_{\sigma}$, we define $\phi\left(\sigma_{1}, \sigma_{2}\right)$ to be $K\left(\mu_{\sigma_{1}}, \mu_{\sigma_{2}}\right)$. The first property follows immediately from the bilinearity of $K$; the second results from the continuity of $K$ as follows:

$$
\begin{aligned}
& \left|\sum_{i, j} \epsilon_{1 i} \phi\left(\sigma_{1 i}, \sigma_{2 j}\right) \epsilon_{2 j}\right|=\left|K\left(\sum_{i} \epsilon_{1 i} \mu_{\sigma_{1 i}}, \quad \sum_{j} \epsilon_{2 j} \mu_{\sigma_{2 i}}\right)\right| \\
& \leqq M(K)\left\|\sum_{i} \epsilon_{1 i} \mu_{\sigma_{1 i}}\right\|_{1}\|\| \sum_{j} \epsilon_{2 j} \mu_{\sigma_{2 j} j} \|_{1} \leqq M(K) ;
\end{aligned}
$$

furthermore, if $K$ is continuous on $\mathfrak{M}_{2} \mathfrak{M}_{2}$, then $\left|\phi\left(\sigma_{1}, \sigma_{2}\right)\right|$ $=\left|K\left(\mu_{\sigma_{1}}, \mu_{\sigma_{2}}\right)\right| \leqq M(K)\left\|\mu_{\sigma_{1}}\right\|_{2} \cdot\left\|\mu_{\sigma_{2}}\right\|_{2}=0$ if $m \sigma_{1}=0$ or $m \sigma_{2}=0$. It follows from inequality $(5)$ that $T \phi \leqq M(K)$ and by the usual argument that the equality holds.

Let $\mu_{1}, \mu_{2}$ be any two bounded, measurable functions. Denoting by $\mu_{1 m}$ and $\mu_{2 n}$ the sums

$$
\sum_{i=0}^{m-1} d_{1 i} \mu_{\sigma_{1 i}} \quad \text { and } \quad \sum_{j=0}^{n-1} d_{2 j} \mu_{\sigma_{2 j}},
$$

where $d_{k i}$ and $\sigma_{k i}$ have the same significance as before, we see that

$$
K\left(\mu_{1 m}, \mu_{2 n}\right)=\sum_{i, j} d_{1 i} d_{2 j} \phi\left(\sigma_{1 i}, \sigma_{2 j}\right) ;
$$

the result then follows by the continuity of $K$.

It is of some interest to note that when $\phi$ has the properties enumerated above, the functional defined by equation (4) is linear and continuous.

5. The Iterated Integrals. To establish the existence of the iterated integrals it will be shown that the Radon integral

$$
\Phi(\sigma)=\int_{0}^{1} \mu_{2}(t) \phi(\sigma, d \Im)
$$


exists, is additive, and is of limited variation. To do this let $\sigma_{c}$ be the complement of $\sigma$ and $\sigma_{1}, \cdots, \sigma_{n}$ be any division of $\Im$ into disjoint sets. Then, if $\phi$ is of limited variation, we have

$$
\left|e \sum_{j=1}^{n} \phi\left(\sigma, \sigma_{j}\right) \epsilon_{j}+e_{c} \sum_{j=1}^{n} \phi\left(\sigma_{c}, \sigma_{j}\right) \epsilon_{j}\right| \leqq T \phi ;
$$

hence taking $\epsilon_{j}$ to be the sign of $\phi\left(\sigma, \sigma_{j}\right), e$ to be 1 , and $e_{c}$ the sign of $\sum \phi\left(\sigma_{c}, \sigma_{j}\right) \epsilon_{j}$, we have

$$
\sum_{j=1}^{n}\left|\phi\left(\sigma, \sigma_{j}\right)\right| \leqq T \phi .
$$

This establishes the existence of $\Phi(\sigma)$, for every $\sigma$. The additivity of $\Phi$ is obvious. Hence we have the following theorem:

THEOREM 3. If $\phi$ on $\subseteq \subseteq 5$ to $\mathfrak{A}$ is bi-additive and of limited variation, then the iterated integrals

$$
\begin{aligned}
& \int_{0}^{1} \mu_{1}(s) \int_{0}^{1} \mu_{2}(t) \phi(d \Im, d \Im) \quad a n d \\
& \int_{0}^{1} \mu_{2}(t) \int_{0}^{1} \mu_{1}(s) \phi(d \Im, d \Im)
\end{aligned}
$$

exist and equal the double integral.

The University of Chicago 